\title{
Definably complete Baire structures
}

by

\author{
Antongiulio Fornasiero (Münster) and Tamara Servi (Lisboa)
}

\begin{abstract}
We consider definably complete Baire expansions of ordered fields: every definable subset of the domain of the structure has a supremum and the domain cannot be written as the union of a definable increasing family of nowhere dense sets. Every expansion of the real field is definably complete and Baire, and so is every o-minimal expansion of a field. Moreover, unlike the o-minimal case, the structures considered form an axiomatizable class. In this context we prove a version of the Kuratowski-Ulam Theorem, some restricted version of Sard's Lemma and a version of Khovanskii's Finiteness Theorem. We apply these results to prove the o-minimality of every definably complete Baire expansion of an ordered field with any family of definable Pfaffian functions.
\end{abstract}

1. Introduction. We recall that a subset $A$ of a topological space $X$ is said to be meager if there exists a collection $\left\{Y_{i}: i \in \mathbb{N}\right\}$ of nowhere dense subsets of $X$ such that $A \subseteq \bigcup_{i \in \mathbb{N}} Y_{i}$. The Baire Category Theorem implies that every open subset of $\mathbb{R}$ (with the usual topology) is nonmeager, i.e. $\mathbb{R}$ is a Baire space.

The notion of Baire space is clearly not first order. Here we consider a similar (definable) notion, which instead is preserved under elementary equivalence, and which coincides with the classical notion over the real numbers (this is made precise in Subsection 2.2.

The (first order) structures we consider are definably complete expansions of ordered fields. Definable completeness (see Definition 2.1) is a weak version of Dedekind completeness, which is preserved under elementary equivalence.

It is shown in [17, [21], [10] that, as in the o-minimal case, (a definable version of) most results of elementary real analysis can be proved in every definably complete expansion of an ordered field. However, to obtain less elementary results one would need some more sophisticated machinery, in the

2010 Mathematics Subject Classification: Primary 58A17; Secondary 03C64, 32C05, $54 \mathrm{E} 52$.

Key words and phrases: Pfaffian functions, definably complete structures, Baire spaces, o-minimality. 
direction of Sard's Lemma and Fubini's Theorem. Both of the cited classical results refer to a notion of smallness (having measure zero), which has no natural translation in our context. We consider instead a topological notion of smallness (being meager), propose a definable version of this notion and carry out a theory of definably complete Baire (DCB) structures, i.e. expansions of ordered fields such that every definable subset of the domain has a supremum and the domain cannot be written as the union of a definable increasing family of nowhere dense sets.

One of our aims in this article is to extend a few standard facts from real analysis to the "definable" context: for DCB structures we prove an analogue to Fubini's Theorem (the Kuratowski-Ulam Theorem 4.1) and a weak form of Sard's Lemma (Theorem 6.8). We claim that these results are strong enough to extend some nontrivial theorems to DCB structures: as a test case, we show that, in analogy with Khovanskii's theory [14, there are uniform and recursive bounds on the number of definably connected components of definable Pfaffian varieties (Definition 7.1 and Theorem 7.2).

Our second aim is to give new examples of definably complete structures, o-minimal or not (see Subsection 2.3); this theme will be developed more fully in [7].

Our third aim is to apply the theorems about "abstract" DCB structures to obtain new results about o-minimal expansion of the real line. An example is given in [9], where we show the existence of recursive bounds on some topological invariants of sets definable in the Pfaffian closure of an o-minimal expansion of the real field.

Every o-minimal expansion of a field is a definably complete Baire structure (see 2.17). The converse is clearly not true. However, in Section 8 we give necessary and sufficient conditions for a definably complete Baire expansion of a field by $\mathcal{C}^{\infty}$ functions to be o-minimal (Theorem 8.1). We derive the o-minimality of every definably complete Baire expansion of an ordered field with any family of definable Pfaffian functions (e.g. an exponential function). For instance, let $\mathbb{K}$ be an ordered exponential field (i.e. an expansion of an ordered field with a function $f$ satisfying $\left.f^{\prime}=f, f(0)=1\right)$. Then our results imply that $\mathbb{K}$ is o-minimal iff $\mathbb{K}$ is definably complete and Baire (see also Remark 8.3).

Finally, a few words about the proofs. One of the main difficulties to extend results from the real case to the nonarchimedean context is that the union of a countable family of definable sets is not necessarily definable. Therefore, we have to modify the definitions and proofs that involve countable unions: one cogent example is in the definition of meager sets (and therefore of DCB structures: see Subsection 2.2). While sometimes we are successful in these modifications, other times we have to settle for weaker 
results: see the discussion after Corollary 3.9, or consider the fact that we are able to prove Sard's Lemma only under a stronger hypothesis than usual (Subsection 6.1). Another difficulty (which however does not appear in this article) is that we cannot construct sets by recursion: an important example is given by the proof that the real line is not meager, which we are still not able to generalize to definably complete structures (Conjecture 2.20).

1.1. Notation. Throughout this paper, $\mathbb{K}$ is a (first-order) structure expanding an ordered field. We use the word "definable" as a shorthand for "definable in $\mathbb{K}$ with parameters from $\mathbb{K}$ ".

We denote by $x, y, z, \ldots$ the points in $\mathbb{K}^{n}$. When we want to stress the fact that they are tuples, we write $\bar{x}, \bar{y}, \bar{z}, \ldots$, where $\bar{x}=\left(x_{1}, \ldots, x_{n}\right)$, etc.

For convenience, on $\mathbb{K}^{m}$ instead of the usual Euclidean distance we will use the equivalent distance

$$
d:(x, y) \mapsto \max _{i=1, \ldots, m}\left|x_{i}-y_{i}\right| .
$$

For $\delta>0$ and $x \in \mathbb{K}^{m}$, we denote by $B^{m}(x ; \delta):=\left\{y \in \mathbb{K}^{m}: d(x, y)<\delta\right\}$ the open "ball" of center $x$ and "radius" $\delta$ and its closure by $\bar{B}^{m}(x ; \delta)$; we will drop the superscript $m$ if it is clear from the context.

We denote by $\tilde{\mathbb{R}}$ the expansion of the real field with a predicate for every subset of $\bigcup_{n \in \mathbb{N}} \mathbb{R}^{n}$.

Let $X \subseteq Y \subseteq \mathbb{K}^{n}$, with $Y$ definable. We write $\operatorname{cl}_{Y}(X)$ (or simply $\bar{X}$ if $Y$ is clear from the context) for the topological closure of $X$ in $Y$, $\operatorname{int}_{Y}(X)$ (or simply $X$ ) for the interior of $X$ in $Y, \operatorname{bd}_{Y}(X):=\bar{X} \backslash X$ for the boundary of $X$ (in $Y$ ), and $\partial_{Y} X:=\bar{X} \backslash X$ for the frontier of $X$ (in $Y$ ).

We define $\Pi_{n}^{m+n}: \mathbb{K}^{m+n} \rightarrow \mathbb{K}^{m}$ to be the projection onto the first $m$ coordinates. If $A \subset \mathbb{K}^{m+n}$ and $x \in \mathbb{K}^{m}$, we denote by $A_{x}$ the fibre of $A$ over $x$, i.e. the set $\left\{y \in \mathbb{K}^{n}:(x, y) \in A\right\}$.

\section{Definably complete Baire structures}

\subsection{Definably complete structures}

Definition 2.1. An expansion $\mathbb{K}$ of an ordered field is called definably complete if every definable subset of $\mathbb{K}$ has a supremum in $\mathbb{K} \cup\{ \pm \infty\}$.

Generalities on definably complete structures (first introduced in [20]) can be found in [21], [4, §2], [10], and [17].

From now on, $\mathbb{K}$ will denote a definably complete expansion of an ordered field.

Definition 2.2. $X \subseteq \mathbb{K}^{m}$ is definably compact (d-compact for short) if it is definable, closed in $\mathbb{K}^{m}$, and bounded. 
Definably compact sets were introduced (for $\mathbb{K}$ o-minimal) in [19]. We will often use without further comment the following results:

Lemma 2.3 ([17]). Let $X \subseteq \mathbb{K}^{n}$ be definable. Then $X$ is definably compact iff for every definable decreasing family $(Y(t))_{t \in \mathbb{K}}$ of closed nonempty subsets of $X$, we have $\bigcap_{t} Y(t) \neq \emptyset$.

Lemma 2.4 (17]). Let $f: \mathbb{K}^{n} \rightarrow \mathbb{K}^{m}$ be a definable continuous function and let $C \subset \mathbb{K}^{n}$ be d-compact. Then $f(C)$ is d-compact.

Definition 2.5. An n-dimensional definable embedded $\mathcal{C}^{N} \mathbb{K}$-manifold $V \subseteq \mathbb{K}^{d}$ (which we will simply call $n$-dimensional $\mathbb{K}$-manifold) is a definable subset $V$ of $\mathbb{K}^{d}$ such that for every $x \in V$ there exists a definable neighbourhood $U(x)$ of $x$ (in $\mathbb{K}^{d}$ ) and a definable $\mathcal{C}^{N}$ diffeomorphism $f_{x}: U(x) \simeq \mathbb{K}^{d}$ such that $U(x) \cap V=f_{x}^{-1}\left(\mathbb{K}^{n} \times\{0\}\right)$.

REMARK 2.6. Note that a $\mathbb{K}$-manifold $V$ can always be written as the intersection of a definable closed set and a definable open set. In fact, let $\delta: V \rightarrow \mathbb{K} \cup\{+\infty\}$ be the definable map

$\delta(x):=\sup \{r \in \mathbb{K}: \forall s \in \mathbb{K}(0<s<r \Rightarrow B(x ; s) \cap V$ is closed in $B(x ; s))\}$. Let $U:=\bigcup_{x \in V} B(x ; \delta(x) / 2)$; then $V=\bar{V} \cap U$.

Note moreover that the dimension $n$ of a $\mathbb{K}$-manifold $V$ is uniquely determined by $V$, because $\mathbb{K}^{n}$ and $\mathbb{K}^{n^{\prime}}$ are locally diffeomorphic iff $n=n^{\prime}$. If we consider only $\mathcal{C}^{0}$ manifolds, it is not clear anymore if the dimension is well defined.

The analogue of the Implicit Function Theorem and its usual corollaries hold in definably complete structures [21].

Finally, recall the following definition.

Definition 2.7. A definable set $X \subset \mathbb{K}^{n}$ is definably connected if it cannot be expressed as a union of two definable nonempty disjoint open sets. A subset $C \subseteq X$ is a definably connected component of $X$ if it is a maximal definably connected subset of $X$.

Note that if $X$ has finitely many definably connected components, then each component of $X$ is definable (it is an atom of the finite Boolean algebra of clopen definable subsets of $X$ ). Moreover, if $\mathbb{K}$ expands the real field, every definable and (topologically) connected set is also definably connected. The converse could in general not be true (but we do not have a counterexample). However it is true if $\mathbb{K}$ is o-minimal.

2.2. Meager sets. Let $X \subseteq Y \subseteq \mathbb{K}^{n}$, with $Y$ definable.

Definition 2.8. $X$ is nowhere dense (in $Y$ ) if $\operatorname{int}_{Y}\left(\operatorname{cl}_{Y}(X)\right)=\emptyset . X$ is definably meager (in $Y$ ) if there exists a definable increasing family $(A(t))_{t \in \mathbb{K}}$ of nowhere dense subsets of $Y$ such that $X \subseteq \bigcup_{t} A(t)$. We will call the family 
$\left(\mathrm{cl}_{Y}(A(t))\right)_{t \in \mathbb{K}}$ a witness of the fact that $X$ is definably meager. $X$ is definably residual (in $Y$ ) if $Y \backslash X$ is definably meager.

Notice that if $(A(t))_{t \in \mathbb{K}}$ is a witness of the fact that $X$ is meager in $\mathbb{K}^{n}$, then also the family

$$
\left(\bar{B}^{n}(0 ;|t|) \cap A(t)\right)_{t \in \mathbb{K}}
$$

is a witness, hence we may always assume that each $A(t)$ is d-compact.

Notice also that we do not require that a definably meager set is definable.

The subsets of $Y$, with the operations $\triangle$ (symmetric difference) and $\cap$, form a commutative ring; the definably meager subsets of $Y$ form an ideal of this ring.

Definition 2.9. $Y$ is definably Baire if no nonempty open definable subset of $Y$ is definably meager (in $Y$ ).

Note that if $\mathbb{K}$ has countable cofinality, then $X$ is definably meager (Baire, respectively) in $\mathbb{K}^{n}$ if $X$ is meager (Baire, respectively) in the usual topological sense. In general, the converse is not true: for instance, if $\mathbb{K}$ is a countable o-minimal structure, then it is definably Baire, but not Baire in the topological sense. However, clearly the two notions coincide for $\tilde{\mathbb{R}}$.

Convention 2.10. From now on, we will write "meager" for "definably meager", and "topologically meager" for the usual topological notion, and similarly for "residual" and "Baire".

As in the classical case, one can prove the following results.

Proposition 2.11. Let $Y$ be definable, and $\emptyset \neq U \subseteq Y$ be definable and open. Then $U$ is meager in $Y$ iff it is meager in itself.

Corollary 2.12. Let $Y \subseteq \mathbb{K}^{m}$ be definable. The following are equivalent:

(1) $Y$ is Baire;

(2) for all $X \subseteq Y$, if $X$ is meager, then ${ }^{\circ}=\emptyset$;

(3) every $x \in Y$ has a definable neighbourhood (in $Y$ ) which is Baire.

REMARK 2.13. Note that, for all $n \in \mathbb{N}, \mathbb{K}^{n}$ is Baire iff it is not meager in itself. In fact, let $B \subset \mathbb{K}^{n}$ be an open box. If $B$ is meager in $\mathbb{K}^{n}$ then, by Proposition 2.11, $B$ is also meager in itself. Since $\mathbb{K}$ expands a field, $B$ is definably homeomorphic to $\mathbb{K}^{n}$, hence $\mathbb{K}^{n}$ is meager in itself.

The following result is not trivial and will be proved in Section 4

Proposition 2.14. If $\mathbb{K}$ is Baire, then for every $m \geq 1, \mathbb{K}^{m}$ is Baire.

The converse, however, is trivial: if $\mathbb{K}^{m}$ is Baire for some $m \geq 1$, then $\mathbb{K}$ is Baire. 


\subsection{DCB structures: definition and examples}

Definition 2.15. An expansion $\mathbb{K}$ of an ordered field is a definably complete Baire structure (DCB) if it is definably complete and $\mathbb{K}$ is definably Baire as a definable subset of $\mathbb{K}$ itself, in the sense of Def. 2.9. A theory $T$ is definably complete and Baire if every model of $T$ is a definably complete Baire structure.

REMARK 2.16. The fact that $\mathbb{K}$ is definably complete and Baire can be expressed by a first-order axiom scheme; therefore, every $\mathbb{K}^{\prime}$ elementarily equivalent to $\mathbb{K}$ also satisfies the hypothesis.

Moreover, an ultraproduct of definably complete Baire structures is also definably complete and Baire; the same cannot be said for o-minimal structures.

Moreover, a reduct of a definably complete (and Baire) structure, which does still expand an ordered field, is also definably complete (and Baire).

EXAMPLES 2.17. The following are examples of definably complete Baire structures.

- Every expansion of $\mathbb{R}$ (because $\mathbb{R}$ is Dedekind complete and topologically Baire).

- Every o-minimal expansion of a field. In fact, a nowhere dense definable subset of $\mathbb{K}$ is finite, and definable families of finite sets are uniformly finite; hence, the union of a definable increasing family of nowhere dense sets is finite, and cannot coincide with the whole structure.

- Let $\mathcal{B}$ be an o-minimal expansion of a field, and let $A \preccurlyeq \mathcal{B}$ be a dense substructure. Then the expansion $\mathcal{B}_{A}$ of $\mathcal{B}$, generated by adding a unary predicate symbol for $A$, is definably complete and Baire. This follows from the fact that if $X \subseteq \mathcal{B}$ is $\mathcal{B}_{A}$-definable, then its topological closure $\bar{X}$ is $\mathcal{B}$-definable (see [5, Theorem 4]). Hence, $X$ is dense in a finite union of intervals. Moreover, if $X$ is closed and nowhere dense, then it is finite, and, since $\mathcal{B}_{A}$ satisfies the Uniform Finiteness condition (see [5, Corollary 4.5]), the union of a definable increasing family of nowhere dense sets is finite. More generally, as shown in [4, §3.5], any definably complete structure satisfying the Uniform Finiteness condition is definably Baire (one can even show that if $\mathbb{K}$ is definably complete, and every definable closed discrete subset of $\mathbb{K}$ is bounded, then $\mathbb{K}$ is Baire [7]).

EXAMPLE 2.18. For every $n \in \mathbb{N}$, consider the $n$th iterate of the exponential function, defined inductively as $\exp _{1}=\exp$ and $\exp _{n+1}=\exp \left(\exp _{n}\right)$. Let $\mathcal{L}$ be the language of ordered field with an extra unary function symbol $f$. For each $n \in \mathbb{N}$, let $\mathbb{R}\left(\exp _{n}\right)$ be the expansion of the real field to the $\mathcal{L}$-structure where $f$ is interpreted as $\exp _{n}$. Let $M(f)$ be some nonprincipal ultraproduct of $\left(\mathbb{R}\left(\exp _{n}\right): n \in \mathbb{N}\right)$. It is not known whether $M(f)$ is 
o-minimal (if it were, it would provide the first example of an o-minimal expansion of a field which is not exponentially bounded).

However, since each $\mathbb{R}\left(\exp _{n}\right)$ is o-minimal, we can conclude that $M(f)$ is definably complete and Baire.

EXAMPLE 2.19. Let No be the field of surreal numbers (see e.g. [11]). As shown in [11, 6], No has a canonical structure as an elementary extension of $\mathbb{R}_{a n}(\exp )$, which we denote by $\mathrm{No}(a n, \exp )$, and therefore it is o-minimal. Moreover, since No is isomorphic to the field of power series $\mathbb{R}((\mathrm{No}))$, it also has a full real analytic $\mathbb{R}((\mathbb{Z})$ )-structure, which is also o-minimal (see [3] for the definition and proof), which we denote by $\mathrm{No}(o v)$. We conjecture that the full expansion $\mathrm{No}(a n, \exp , o v)$ is definably complete and Baire. If this were the case, then we claim that this structure would also be o-minimal.

Finally, a general conjecture:

Conjecture 2.20. Every definably complete expansion of a field is also definably Baire.

We have a partial result in this direction:

Proposition 2.21 ([7, Theorem 7.32]). If $\mathbb{K}$ is a definably complete expansion of a field, $Z \subset \mathbb{K}^{n}$ is definable and discrete, and $f: Z \rightarrow \mathbb{K}$ is some definable function, then $f$ is not surjective.

3. $\mathcal{F}_{\sigma}$-sets. We now consider a class of sets for which it is easy to determine whether they are meager or not. These sets have also been studied in [4, where they are called $D_{\Sigma}$-sets.

Definition 3.1. Let $X \subseteq Y \subseteq \mathbb{K}^{n}$, with $Y$ definable. $X$ is in $\mathcal{F}_{\sigma}$ in $Y$ (or " $X$ is an $\mathcal{F}_{\sigma}$-subset of $Y$ ") if $X$ is the union of a definable increasing family of closed subsets of $Y$, indexed by $\mathbb{K} . X$ is in $\mathcal{G}_{\delta}$ if its complement is in $\mathcal{F}_{\sigma}$.

The proof of the following lemma can be found in [4].

Lemma 3.2. Let $\mathcal{A}$ be either the family of $\mathcal{F}_{\sigma}$-subsets or the family of $\mathcal{G}_{\delta}$-subsets of $\mathbb{K}^{n}$, for some $n \in \mathbb{N}$. Then each $A \in \mathcal{A}$ is definable. $\mathcal{A}$ is closed under finite unions, finite intersections, Cartesian products and preimages under definable continuous functions. Finite Boolean combinations of definable open subsets of $\mathbb{K}^{n}$ are in $\mathcal{A}$. The family of $\mathcal{F}_{\sigma}$-subsets is also closed under images under definable continuous functions.

In general, the family of $\mathcal{G}_{\delta}$-subsets of some $\mathbb{K}^{n}$ is not closed under images under definable continuous functions.

Notice that, by Remark 2.6, every $\mathbb{K}$-manifold is an $\mathcal{F}_{\sigma}$-set.

Remark 3.3. Let $X \subseteq \mathbb{K}^{n}$. Then $X$ is in $\mathcal{F}_{\sigma}$ iff $X$ is of the form $\Pi_{n}^{n+m}(Z)$ for some $Z \subseteq \mathbb{K}^{n+m}$ closed and definable. In fact, let $(X(t))_{t \in \mathbb{K}}$ 
be a definable increasing family of closed subsets of $\mathbb{K}^{n}$ such that $X=$

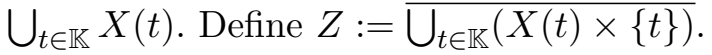

Notice that if $\mathbb{K}$ is o-minimal, then every definable subset $X$ of $\mathbb{K}$ is a finite Boolean combination of definable closed sets (because $X$ is a finite union of cells), and therefore $X$ is in $\mathcal{F}_{\sigma}$.

REMARK 3.4. If $X \subseteq \mathbb{K}^{n}$ is meager, then there exists a meager $\mathcal{F}_{\sigma}$-set containing $X$.

The main property of $\mathcal{F}_{\sigma}$-sets is the following.

Lemma 3.5. Let $Y$ be definable and Baire, and $D \subseteq Y$. Assume that $D$ is in $\mathcal{F}_{\sigma}$. Then $D$ is meager iff $\stackrel{D}{D}=\emptyset\left(\left(^{1}\right)\right.$.

Proof. If $\stackrel{\circ}{D} \neq \emptyset$, then, since $Y$ is Baire, $D$ cannot be meager. Conversely, assume that $D$ is not meager. If $D$ is in $\mathcal{F}_{\sigma}$, then $D=\bigcup_{t} D(t)$ for some definable increasing family of closed subsets. Since $D$ is not meager, at least one of the $D(t)$, say $D\left(t_{0}\right)$, satisfies $\operatorname{int}\left(D\left(t_{0}\right)\right) \neq \emptyset$ (otherwise, $D\left(t_{0}\right)$ would be nowhere dense), and therefore $\stackrel{D}{D} \neq \emptyset$.

Note that if $X \subseteq \mathbb{R}^{n}$ is in $\mathcal{F}_{\sigma}$ for the previously mentioned structure $\tilde{\mathbb{R}}$, and has Lebesgue measure zero, then $X$ is meager, but the converse is not true.

We now give a local condition which is sufficient to prove that the image of an $\mathcal{F}_{\sigma}$-set under a continuous definable function is meager.

Proposition 3.6. Let $C \subseteq \mathbb{K}^{m} \times \mathbb{K}^{n}$ be in $\mathcal{F}_{\sigma}, f: C \rightarrow \mathbb{K}^{d}$ be definable and continuous. Assume that for every $y \in \Pi_{m}^{m+n}(C)$ there exists a neighbourhood $V_{y} \subseteq \mathbb{K}^{m}$ of $y$, such that $f\left(\left(V_{y} \times \mathbb{K}^{n}\right) \cap C\right)$ is meager. Then $f(C)$ is meager.

The next lemma will be used in the proof of the above proposition.

Lemma 3.7. Let $C \subset \mathbb{K}^{n}$ be a nonempty d-compact set, and let $V:=$ $\{V(t): t \in I\}$ be a definable open cover of $C$. Then there exists $0<\delta_{0} \in \mathbb{K}$ ( $a$ Lebesgue number for $V$ and $C$ ) such that, for every subset $X \subseteq C$ of diameter smaller than $\delta_{0}$, there exists $t \in I$ such that $X \subseteq V(t)$.

Proof. Suppose for a contradiction that $(\forall \delta>0)(\exists y \in C)(\forall t \in I) B(y ; \delta)$ $\nsubseteq V(t)$. For every $\delta>0$, define $Y(\delta):=\{y \in C:(\forall t \in I) B(y ; \delta) \nsubseteq I(t)\}$. Note that $(Y(\delta))_{\delta>0}$ is a definable family of subsets of $C$, increasing as $\delta$ decreases. Let $y_{0}$ be an accumulation point for the family $(Y(\delta))_{\delta>0}$ as $\delta \rightarrow 0$ (which exists by Lemma 2.3).

$\left({ }^{1}\right)$ This is not true for $\mathcal{G}_{\delta}$-sets: for instance, the set of irrational numbers in $\tilde{\mathbb{R}}$ is a $\mathcal{G}_{\delta}$ which is not meager (it is even residual), but has empty interior. 
Let $t_{0} \in I$ and $\delta_{0}>0$ be such that $B\left(y_{0} ; 2 \delta_{0}\right) \subseteq V\left(t_{0}\right)$. Let $\delta_{1} \leq \delta_{0}$ be such that there exists $y \in Y\left(\delta_{1}\right)$ with $\left|y-y_{0}\right|<\delta_{0}$. Then $B\left(y ; \delta_{0}\right) \subseteq$ $B\left(y_{0} ; 2 \delta_{0}\right) \subseteq V\left(t_{0}\right)$, contradicting the fact that $y \in Y\left(\delta_{1}\right)$.

Proof of Proposition 3.6. If $\mathbb{K}$ is meager in itself, then by Proposition 2.14 there is nothing to prove. Thus, we may assume that $\mathbb{K}$ (and hence $\mathbb{K}^{d}$ ) is Baire.

We proceed by induction on $m$. The case $m=0$ is clear.

Assume that we have already proved the conclusion for $m-1$ (and every $n$ ). We want to prove it for $m$. First, we consider the case when $C$ is d-compact. We can assume that $0 \in C$. Remember that, for every $r>0$ and $y \in \mathbb{K}^{m}, \bar{B}^{m}(y ; r) \subset \mathbb{K}^{m}$ is the closed hypercube of side $2 r$ and centre $y$; let $S^{m}(y ; r)$ be its boundary. Moreover, define $D(r):=f\left(C \cap\left(\bar{B}^{m}(0 ; r) \times \mathbb{K}^{n}\right)\right)$.

Note that $f(C)=\bigcup_{r} D(r)$ and each $D(r)$ is d-compact. Therefore, to prove that $f(C)$ is meager, it suffices to prove that each $D(r)$ has empty interior. Suppose, for a contradiction, that $f(C)$ is not meager, and let

$$
r_{0}:=\inf \{r>0: \operatorname{int}(D(r)) \neq \emptyset\} .
$$

Since the $D(r)$ are closed, $r_{0}=\inf \{r>0: D(r)$ is not meager $\}$. We have $r_{0}>0$ by hypothesis, and $r_{0}<+\infty$ because $f(C)$ is not meager.

Let $P:=\Pi_{m}^{n+m}(C)$. Since $P$ is d-compact, if $\mathbb{K}=\mathbb{R}$, we could find $y_{1}, \ldots, y_{k} \in P$ such that $P \subseteq V_{y_{1}} \cup \cdots \cup V_{y_{k}}$. In the general situation, we need another argument. Let $5 \delta_{0}$ be a Lebesgue number for the open cover $\left\{V_{y}: y \in P\right\}$ of $P$ (we may also assume that $\delta_{0}$ is small in comparison with $\left.r_{0}\right) ; \delta_{0}>0$ exists by Lemma 3.7 .

Note that

$$
\bar{B}^{m}\left(0 ; r_{0}+\delta_{0} / 2\right) \subseteq \bar{B}^{m}\left(0 ; r_{0}-\delta_{0} / 2\right) \cup \bigcup_{y \in S^{m}\left(0 ; r_{0}\right)} \bar{B}^{m}\left(y ; \delta_{0}\right),
$$

hence

$$
D\left(r_{0}+\delta_{0} / 2\right) \subseteq D\left(r_{0}-\delta_{0} / 2\right) \cup \bigcup_{y \in S^{m}\left(0 ; r_{0}\right)} f\left(C \cap\left(\bar{B}^{m}\left(y ; \delta_{0}\right) \times \mathbb{K}^{n}\right)\right) .
$$

By the definition of $r_{0}$, we know that $D\left(r_{0}+\delta_{0} / 2\right)$ is not meager, while $D\left(r_{0}-\delta_{0} / 2\right)$ is meager. Hence, to obtain a contradiction, it suffices to show that $\bigcup_{y \in S^{m}\left(0 ; r_{0}\right)} f\left(C \cap\left(\bar{B}^{m}\left(y ; \delta_{0}\right) \cap \mathbb{K}^{n}\right)\right)$ is meager.

Note that $S^{m}\left(0 ; r_{0}\right)$ is the finite union of the faces of the closed hypercube $\bar{B}^{m}\left(0 ; r_{0}\right)$ : hence, we only need to show that for each face $S$ of $S^{m}\left(0 ; r_{0}\right)$ the set $D:=\bigcup_{y \in S} f\left(C \cap\left(\bar{B}^{m}\left(y ; \delta_{0}\right) \times \mathbb{K}^{n}\right)\right)$ is meager. We can assume that $S$ is the "top" face $\left\{y \in \bar{B}^{m}\left(0 ; r_{0}\right): y_{m}=r_{0}\right\}$ and we may identify $S$ with $\bar{B}^{m-1}\left(0 ; r_{0}\right) \times\left\{r_{0}\right\}$. 
Define

$$
\tilde{C}:=C \cap \bigcup_{y \in S}\left(\bar{B}^{m}\left(y ; \delta_{0}\right) \times \mathbb{K}^{n}\right), \quad \tilde{f}:=f \uparrow \tilde{C} .
$$

Claim. $\tilde{C}$ and $\tilde{f}$ satisfy the hypothesis of the proposition, with $n^{\prime}=n+1$, $m^{\prime}=m-1$, and $V_{z}^{\prime}=B\left(z ; \delta_{0}\right)$.

$\tilde{C}$ is d-compact, and therefore it is in $\mathcal{F}_{\sigma}$. Let $\tilde{P} \subseteq \mathbb{K}^{m-1}$ be the projection of $\tilde{C}$ onto $\mathbb{K}^{m-1}$; note that $\tilde{P}$ is d-compact. Fix $z \in \tilde{P}$; by definition, there exists $t \in\left[r_{0}-\delta_{0}, r_{0}+\delta_{0}\right]$ such that $y:=(z, t) \in P$. Notice that $\tilde{C} \cap\left(V_{z}^{\prime} \times \mathbb{K} \times \mathbb{K}^{n}\right) \subseteq C \cap\left(V_{z}^{\prime} \times\left[r_{0}-\delta_{0}, r_{0}+\delta_{0}\right] \times \mathbb{K}^{n}\right) \subseteq C \cap\left(\bar{B}^{m}\left(y ; 2 \delta_{0}\right) \times \mathbb{K}^{n}\right)$.

Since $5 \delta_{0}$ is a Lebesgue number for the cover $\left\{V_{y}: y \in P\right\}$ of $P$, it follows that there exists $y^{\prime} \in P$ such that $\bar{B}^{m}\left(y ; 2 \delta_{0}\right) \subset V_{y^{\prime}}$. Putting everything together, we find that $\tilde{C} \cap\left(V_{z}^{\prime} \times \mathbb{K}^{n+1}\right) \subset C \cap\left(V_{y^{\prime}} \times \mathbb{K}^{n}\right)$ and thus $\tilde{f}(\tilde{C} \cap$ $\left.\left(V_{z}^{\prime} \times \mathbb{K}^{n+1}\right)\right)$ is meager, which proves the claim.

Therefore, by inductive hypothesis, $\tilde{f}(\tilde{C})$ is meager. However, $D \subseteq \tilde{f}(\tilde{C})$, and we have reached a contradiction.

We now treat the general case when $C$ is in $\mathcal{F}_{\sigma}$. Note that $C$ is an increasing union of d-compact sets $C(t)$. For each $t \in \mathbb{K}$, define $D(t):=$ $f(C(t))$; note that each $D(t)$ is d-compact. By the d-compact case, we can conclude that each $D(t)$ is meager, and therefore nowhere dense. Thus, $D=$ $\bigcup_{t} D(t)$ is meager.

Corollary 3.8. Let $C \subseteq \mathbb{K}^{m}$ be in $\mathcal{F}_{\sigma}$, and $f: C \rightarrow \mathbb{K}^{d}$ be definable and continuous. Assume that for every $x \in C$ there exists a neighbourhood $V_{x}$ of $x$ such that $f\left(C \cap V_{x}\right)$ is meager. Then $f(C)$ is meager.

Proof. Apply the proposition to the case $n=0$.

Corollary 3.9. Let $C \subseteq \mathbb{K}^{m}$ be in $\mathcal{F}_{\sigma}$. If every $x \in C$ has a neighbourhood $V_{x}$ such that $C \cap V_{x}$ is meager, then $C$ is meager.

Proposition 3.6 and the following corollaries are trivial when $\mathbb{K}$ is ominimal, since in this case $C \subset \mathbb{K}^{n}$ is meager iff $\operatorname{dim}(C)<n$.

For the classical topological notions, we know the following facts to be true:

(1) Let $C \subseteq \mathbb{R}^{n}$ and $f: C \rightarrow Y$ (not necessarily continuous). Assume that, for every $x \in C$, there exists a neighbourhood $V_{x} \subseteq C$ of $x$ such that $f\left(C \cap V_{x}\right)$ is topologically meager. Then $f(C)$ is topologically meager.

(2) Let $C \subseteq Y$. If every $x \in C$ has a neighbourhood $V_{x}$ such that $V_{x} \cap C$ is topologically meager, then $C$ is topologically meager.

The first fact follows from the fact that $\mathbb{R}^{n}$ is second countable; the second from [13, Theorem 6.35]. We have been able to prove the definable 
versions only under an additional hypothesis (e.g., $C$ in $\mathcal{F}_{\sigma}$ ); however, these results are strong enough for our applications.

Lemma 3.10. Let $f: \mathbb{K}^{n} \rightarrow \mathbb{K}^{m}$ be definable, and

$$
\mathcal{D}_{f}:=\{\bar{x} \in A: f \text { is discontinuous at } \bar{x}\} \text {. }
$$

If the graph of $f$ is an $\mathcal{F}_{\sigma}$-set, then $\mathcal{D}_{f}$ is meager.

Proof. If, for a contradiction, $\mathcal{D}_{f}$ is not meager, then since it is an $\mathcal{F}_{\sigma}$, it contains a nonempty open box $B$. Therefore, we can assume that $\mathcal{D}_{f}=\mathbb{K}^{n}$, and that $\mathbb{K}^{n}$ is Baire. Let $\Gamma(f)=\bigcup_{t} X(t)$, where $(X(t))_{t \in \mathbb{K}}$ is a definable increasing family of d-compact sets. Let $Y(t):=\Pi_{n}^{n+m}(X(t))$. Note that each $Y(t)$ is d-compact, and $\mathbb{K}^{n}=\bigcup_{t} Y(t)$. Since $\mathbb{K}^{n}$ is Baire, there exists $t_{0}$ such that $Y\left(t_{0}\right)$ contains a nonempty open box $B^{\prime}$. Let $B^{\prime \prime} \subseteq B^{\prime}$ be a closed box with nonempty interior, and $g:=f\left\lceil B^{\prime \prime}\right.$. Note that $\Gamma(g)=$ $X\left(t_{0}\right) \cap\left(B^{\prime \prime} \times \mathbb{K}^{m}\right)$; therefore, $\Gamma(g)$ is d-compact, and so, as in the classical case, $g$ is continuous, contradicting the fact that $B^{\prime \prime} \subseteq \mathcal{D}_{f}$.

4. The Kuratowski-Ulam Theorem. The main result of this section is the following theorem.

Theorem 4.1. Let $D \subseteq \mathbb{K}^{m+n}$. Define $T:=T^{m}(D):=\left\{x \in \mathbb{K}^{m}\right.$ : $D_{x}$ is meager in $\left.\mathbb{K}^{n}\right\}$. If $D$ is meager (in $\mathbb{K}^{m+n}$ ), then $T$ is residual.

This is a definable version of the Kuratowski-Ulam Theorem [18, Theorem 15.1], which in turn is an analogue of Fubini's Theorem: they both imply that if $D$ is negligible, then $D_{y}$ is negligible for almost every $y$; in the Kuratowski-Ulam Theorem negligible means "meager", while in Fubini's Theorem negligible means "of measure zero".

It is not clear whether in the above theorem $D$ definable implies that $T$ is definable. Note that if $\mathbb{K}$ is o-minimal and $D$ is definable, then $T$ is also definable.

As a corollary, we obtain Proposition 2.14

Proof of Proposition 2.14. By induction on $m$. The case $m=1$ is our assumption on $\mathbb{K}$. Assume that we have already proved that $\mathbb{K}^{m}$ is Baire: we want to prove that $\mathbb{K}^{m+1}$ is Baire. Suppose not; then $\mathbb{K}^{m+1}$ is meager in itself. If we apply Theorem 4.1 with $n=1$, we find that either $\mathbb{K}^{m}$ or $\mathbb{K}$ is meager in itself, a contradiction.

Definition 4.2. A definable function $f: Y \rightarrow \mathbb{K}$ is lower semi-continuous if, for every $x \in Y$, either $x$ is an isolated point of $Y$, or

$$
\liminf _{\substack{x^{\prime} \rightarrow x \\ x^{\prime} \in Y}} f\left(x^{\prime}\right) \geq f(x) \text {. }
$$

Remark 4.3. Let $C \subseteq \mathbb{K}^{n+1}$ be d-compact. For every $x \in D:=\Pi_{n}^{n+1}(C)$, let $f(x):=\min C_{x}$. Then $f: D \rightarrow \mathbb{K}$ is lower semi-continuous. 
Lemma 4.4. Let $Y \subseteq \mathbb{K}^{n}$ be definable, $f: Y \rightarrow \mathbb{K}$ be lower semicontinuous and definable, and $\mathcal{D}_{f} \subseteq Y$ be the set of points of discontinuity of $f$. Then $\mathcal{D}_{f}$ is meager (in $Y$ ).

Proof. See [4, Lemma 2.8(1)].

In the above lemma, if $Y=\mathbb{K}=\mathbb{R}$, we cannot conclude that $\mathcal{D}_{f}$ has Lebesgue measure zero. In fact, let $C \subseteq \mathbb{R}$ be closed, with empty interior, and of positive measure, and $f$ be the characteristic function of $\mathbb{R} \backslash C$. Then $\mathcal{D}_{f}=C$, and therefore it is of positive measure.

On the other hand, it is always true that if $f: \mathbb{K}^{m} \rightarrow \mathbb{K}$ is definable, then $\mathcal{D}_{f}$ is in $\mathcal{F}_{\sigma}$ (see the proof of [18, Theorem 7.1]).

Proof of Theorem 4.1. If $\mathbb{K}^{m}$ is meager in itself, then the conclusion is trivially true, because then every subset of $\mathbb{K}^{m}$ is meager. Hence, we can assume that $\mathbb{K}^{m}$ is Baire.

CASE 1: $n=1$ and $D$ is d-compact. Hence, $D$ has empty interior, and each $D_{x}$ is also d-compact. Therefore, by Lemma 3.5. $T=\left\{x \in \mathbb{K}^{m}: D_{x}=\emptyset\right\}$. Let $E:=\mathbb{K}^{m} \backslash T$. We have to prove that $E$ is meager.

For every $\varepsilon>0$ let $X(\varepsilon):=\left\{(x, y) \in \mathbb{K}^{m} \times \mathbb{K}: B^{1}(y ; \varepsilon) \subseteq D_{x}\right\}$. Let $E(\varepsilon):=\pi(X(\varepsilon))=\left\{x \in \mathbb{K}^{m}: D_{x}\right.$ contains a ball of radius $\left.\varepsilon\right\}$. Note that $X(\varepsilon)$ is d-compact, since its complement is the projection of an open set, therefore so is $E(\varepsilon)$. Note that $E=\bigcup_{\varepsilon>0} E(\varepsilon)$; hence, to prove that $E$ is meager, it suffices to prove that each $E(\varepsilon)$ is nowhere dense. Since each $E(\varepsilon)$ is d-compact, it suffices to prove the following claim.

Claim 1. For every $\varepsilon>0, \operatorname{int}(E(\varepsilon))=\emptyset$ (see also [4, Lemma 2.8(2)]).

Assume, for a contradiction, that there exists a nonempty open box $U \subseteq E(\varepsilon)$. Define

$$
f: U \rightarrow \mathbb{K}, \quad x \mapsto \min \{y \in \mathbb{K}:(x, y) \in X(\varepsilon)\} .
$$

Note that $f$ is lower semi-continuous and definable. By Lemma 4.4, $f$ is continuous outside a meager set $\mathcal{D}_{f} \subseteq U$. Since $\mathbb{K}^{m}$ is Baire, $\mathcal{D}_{f} \neq U$, and therefore there exists $x_{0} \in U$ such that $f$ is continuous at $x_{0}$. It is now easy to show that a neighbourhood of $\left(x_{0}, f\left(x_{0}\right)\right)$ is contained in $D$, contradicting the fact that $\stackrel{\circ}{D}=\emptyset$.

CASE 2: $n=1$ and $D$ is an arbitrary meager subset of $\mathbb{K}^{m+1}$. Let $(D(p))_{p \in \mathbb{K}}$ be an increasing definable family of d-compact subsets of $\mathbb{K}^{m+1}$ with empty interior such that $D \subseteq \bigcup_{p} D(p)$. For each $p \in \mathbb{K}$, let $E(p):=$ $\left\{x \in \mathbb{K}^{m}: D(p)_{x}\right.$ is not meager in $\left.\mathbb{K}\right\}$. By what we have seen above, $E(p)=$ $\bigcup_{\varepsilon>0} E(p, \varepsilon)$, where $(E(p, \varepsilon))_{0<\varepsilon \in \mathbb{K}, p \in \mathbb{K}}$ is a definable family of subsets of $\mathbb{K}$, increasing in $p$ and decreasing in $\varepsilon$, such that each $E(p, \varepsilon)$ is closed and 
nowhere dense. Let

$$
E^{\prime}:=\bigcup_{\varepsilon, p} E(p, \varepsilon)=\bigcup_{p} E(p) .
$$

Claim 2. $\mathbb{K}^{m} \backslash T \subseteq E^{\prime}$.

In fact, let $x \notin T$. Thus, $D_{x}$ is not meager. However, $D_{x} \subseteq \bigcup_{p} D(p)_{x}$. Since $\left(D(p)_{x}\right)_{p \in \mathbb{K}}$ is an increasing definable family of closed subsets of $\mathbb{K}$, there exists $p_{0}$ such that $D\left(p_{0}\right)_{x}$ has nonempty interior. Thus, $x \in E\left(p_{0}\right) \subseteq E^{\prime}$.

Therefore, it suffices to prove that $E^{\prime}$ is meager to conclude that $T$ is residual. However, $E^{\prime}=\bigcup_{p>0} E(p, 1 / p)$, and we are done.

CASE 3: $n>1$ and $D$ is an arbitrary meager subset of $\mathbb{K}^{m+n}$. We argue by induction on $n$. Suppose that we have already proved the conclusion for $n$ (and for every $m$ ). We want to prove the conclusion for $n+1$. First, we will assume that $D \subseteq \mathbb{K}^{m+n+1}$ is in $\mathcal{F}_{\sigma}$. We want to prove that the set $T:=T^{m}(D):=\left\{x \in \mathbb{K}^{m}: D_{x}\right.$ is meager $\}$ is residual. Define

$$
\begin{aligned}
& S:=\mathbb{K}^{m+1} \backslash T^{m+1}(D):=\left\{\left(x, y_{n+1}\right) \in \mathbb{K}^{m} \times \mathbb{K}: D_{\left(x, y_{n+1}\right)} \text { is not meager }\right\}, \\
& R:=T^{m}(S)=\left\{x \in \mathbb{K}^{m}: S_{x} \text { is meager }\right\} .
\end{aligned}
$$

Notice that (for the moment) we do not know whether $S$ and $R$ are definable, even assuming that $D$ is in $\mathcal{F}_{\sigma}$.

Claim 3. $S$ is meager.

By inductive hypothesis.

Claim 4. $R$ is residual.

By the case $n=1$ and the previous claim.

Claim 5. $R \subseteq T$.

Fix $x \in \mathbb{K}^{m}$. Assume that $x \notin T$. We have to prove that $x \notin R$. Define $F:=D_{x} \subseteq \mathbb{K}^{n+1}$. Note that $F$ is in $\mathcal{F}_{\sigma}$; therefore, since $x \notin T, \stackrel{\circ}{F} \neq \emptyset$. Let $U:=U_{1} \times U_{2}$ be a nonempty open box contained in $F, U_{1} \subseteq \mathbb{K}, U_{2} \subseteq \mathbb{K}^{n}$. For every $y_{n+1} \in U_{1}, D_{\left(x, y_{n+1}\right)}=F_{y_{n+1}} \supseteq U_{2}$, and therefore $\left(x, y_{n+1}\right) \in S$. Thus, $U_{1} \subseteq S_{x}$, and $x \notin R$.

Hence, $T$ contains a residual set, and therefore it is residual.

For $D$ arbitrary, let $D^{\prime} \subseteq \mathbb{K}^{m+n}$ be a meager $\mathcal{F}_{\sigma^{-}}$set containing $D$. By the previous case, the corresponding set $T^{\prime}:=T^{m}\left(D^{\prime}\right)$ is residual. Since $T^{\prime} \subseteq T$, we are done.

5. Almost open sets. Let $\mathbb{K}$ be a definably complete Baire structure. Let $Y \subseteq \mathbb{K}^{m}$ be definable. We have seen that the family of meager subsets of $Y$ is an ideal, hence it defines an equivalence relation on the family of subsets of $Y$, given by $X \sim X^{\prime}$ iff $X \triangle X^{\prime}$ is meager. 
Definition 5.1. $X \subseteq Y$ is almost open (in $Y$ ), or a.o. for short, if $X$ is equivalent to a definable open set $\left({ }^{2}\right)$.

Lemma 5.2. Let $Y \subseteq \mathbb{K}^{m}$ be definable, and $A$ and $B$ be a.o. subsets of $Y$. Then $A \cap B, A \cup B$ and $Y \backslash A$ are also a.o. Moreover, $\mathcal{F}_{\sigma}$-subsets and $\mathcal{G}_{\delta}$-subsets of $Y$ are a.o. Finally, if $Y_{1}$ and $Y_{2}$ are definable, and $A_{i} \subseteq Y_{i}$ are a.o. for $i=1,2$, then $A_{1} \times A_{2}$ is a.o. in $Y_{1} \times Y_{2}$.

The proof is left as an exercise.

Consequently, $X \subseteq Y$ is a.o. iff it is equivalent to a definable closed subset of $Y$.

REMARK 5.3. Every meager set is a.o., being equivalent to the empty set. Every residual set is also a.o., being equivalent to the ambient space.

The following is a partial converse of Theorem 4.1 .

Proposition 5.4. Let $D$ be an a.o. subset of $\mathbb{K}^{m+n}$, and define $T(D):=$ $\left\{x \in \mathbb{K}^{m}: D_{x}\right.$ is meager $\}$. Then $D$ is meager iff $T(D)$ is residual.

Proof. The "only if" direction is Theorem 4.1. For the other direction, let $U$ be an open set such that $E:=D \triangle U$ is meager. By Theorem 4.1, $T(E)$ is residual. Moreover, since $U_{x}=D_{x} \triangle E_{x}$, we have $T(U) \supseteq T(D) \cap T(E)$, and therefore $T(U)$ is also residual. However, $U$ is open and $\mathbb{K}^{n}$ is Baire; therefore, $T(U)$ is the complement of the projection of $U$ on $\mathbb{K}^{m}$. Since $U$ is open, $T(U)$ is closed. Therefore, $T(U)$ is closed and residual; since $\mathbb{K}^{m}$ is Baire, $T(U)=\mathbb{K}^{m}$. Thus, $U$ is empty, and we are done.

The hypothesis that $D$ is a.o. in the above proposition is necessary: [18, Theorem 15.5] gives an example of a set $E \subseteq \mathbb{R}^{2}$ that is not topologically meager and such that no three points of $E$ are collinear.

6. The Sard property. In this section we investigate some consequences of Sard's Lemma, and prove a version of this lemma in a particular case. Let $\mathbb{K}$ be a definably complete Baire structure.

Definition 6.1. Fix positive natural numbers $d, r, m$. Let $V \subseteq \mathbb{K}^{d}$ be a $\mathbb{K}$-manifold of dimension $n$. Let $f: V \rightarrow \mathbb{K}^{m}$ be a definable $\mathcal{C}^{r}$ function and $\Delta_{f}$ be the set of singular points of $f$. If $\Sigma_{f}:=f\left(\Delta_{f}\right)$ is meager in $\mathbb{K}^{m}$, then we say that $f$ has the Sard property.

REMARK 6.2. If $\mathbb{K}$ is o-minimal, then every $\mathcal{C}^{1}$ definable function $f$ : $V \rightarrow \mathbb{K}^{m}$ has the Sard property [1, Theorem 3.5]. If $\mathbb{K}=\tilde{\mathbb{R}}$ and $f: V \rightarrow \mathbb{K}^{m}$ is as in the above definition with $r>\max \{0, n-m\}$, then $f$ has the Sard property. In fact, by Sard's Lemma, $\Sigma_{f}$ has Lebesgue measure zero, and therefore it has empty interior. Since $\Sigma_{f}$ is in $\mathcal{F}_{\sigma}$, it is also meager.

$\left({ }^{2}\right)$ Almost open sets are called "sets with the property of Baire" in [18. 
Proposition 6.3. Suppose $f: \mathbb{K}^{n} \rightarrow \mathbb{K}^{n}$ has the Sard property, and let $C \subset \mathbb{K}^{n}$ be meager. Then $f(C)$ is meager.

Proof. We may assume that $C \in \mathcal{F}_{\sigma}$, since $C$ is contained in a meager $\mathcal{F}_{\sigma^{-}}$-set. Let $\Lambda:=\mathbb{K}^{n} \backslash \Delta_{f}$ be the set of regular points of $f$. Note that $\Lambda$ is open.

By the Sard property, $f\left(C \cap \Delta_{f}\right)$ is meager. Hence, it suffices to show that $f(C \cap \Lambda)$ is meager. Let $x \in C \cap \Lambda$. Since $x$ is a regular point for $f$, by the Implicit Function Theorem there exists a neighbourhood $V$ of $x$ such that $f$ is a diffeomorphism on $V$; therefore, $f(C \cap V)$ is meager, and, by Corollary 3.8, $f(C \cap \Lambda)$ is meager.

The following lemma is a generalization of Proposition 5.4.

Lemma 6.4. Let $f: \mathbb{K}^{n} \rightarrow \mathbb{K}^{m}$ be a $\mathcal{C}^{1}$ definable function with the Sard property. Let $\Lambda$ be the set of regular points of $f$, and $C \subseteq \mathbb{K}^{n}$ be almost open. For every $\bar{y} \in \mathbb{K}^{m}$, let $F_{\bar{y}}:=f^{-1}(\bar{y}), C_{\bar{y}}:=F_{\bar{y}} \cap C$, and $T:=\left\{\bar{y} \in \mathbb{K}^{m}\right.$ : $C_{\bar{y}}$ is meager in $F_{\bar{y}}$ or $\left.F_{\bar{y}}=\emptyset\right\}$. Then $T$ is residual iff $C \cap \Lambda$ is meager.

Proof. This follows from the Implicit Function Theorem, Proposition 5.4 . and Corollary 3.8 .

6.1. The Sard property and Noetherian differential rings. In this subsection we will show a version of Sard's Lemma for functions belonging to a Noetherian differential ring.

Definition 6.5. Fix $n \in \mathbb{N} \backslash\{0\}$ and a definably connected definable open set $U \subseteq \mathbb{K}^{n}$. Let $\mathcal{C}^{\infty}(U, \mathbb{K})$ be the ring of definable $\mathcal{C}^{\infty}$ functions from $U$ to $\mathbb{K}$. Fix a subring $M \subseteq \mathcal{C}^{\infty}(U, \mathbb{K})$ which is Noetherian and closed under partial derivation, and containing $\mathbb{K}\left[x_{1}, \ldots, x_{n}\right]$. $M$ is called a Noetherian differential ring.

If $G:=\left(g_{1}, \ldots, g_{k}\right) \in M^{k}$, we denote by $V(G)$ the set of zeros of $G$, and by $V^{\text {reg }}(G)$ the set of regular zeros of $G$.

Generalities on Noetherian differential rings of functions over definably complete structures can be found in [21]. In particular, we will need the following result, which states that in a Noetherian differential ring there are no flat functions.

Proposition 6.6. Let $0 \not \equiv g \in M$. Then for every $x \in U$ such that $g(x)=0$, there exist $k \in \mathbb{N}$ and a derivative $\theta$ of order $k$ such that $\theta g(x) \neq 0$.

REMARK 6.7. For $g_{1}, \ldots, g_{k} \in M$, the set $V^{\text {reg }}\left(g_{1}, \ldots, g_{k}\right)$ is a $\mathbb{K}$-manifold and hence is in $\mathcal{F}_{\sigma}$.

In this subsection we prove the following version of Sard's Lemma:

Theorem 6.8. Fix $k, m \in \mathbb{N}, k \leq n$. Let

- $H=\left(h_{1}, \ldots, h_{n-k}\right) \in M^{n-k}$ and $V:=V^{\mathrm{reg}}(H) \neq \emptyset$; 
- $F=\left(F_{1}, \ldots, F_{m}\right) \in M^{m}$ and $f:=F \nmid V$.

Then $f: V \rightarrow \mathbb{K}^{m}$ has the Sard property, i.e. $\Sigma_{f}$ is a meager set $\left(\right.$ in $\left.\mathbb{K}^{m}\right)$.

Proof. We proceed by induction on $\operatorname{dim} V$ and $m$. If $m=0$, there are no singular points. If $\operatorname{dim} V=0$, then $V$ is discrete. In particular, for every $a \in \Delta_{f}$ there exists a neighbourhood $U_{a}$ of $a$ such that $\Delta_{f} \cap U_{a}=\{a\}$. Hence we can apply Corollary 3.8 and we are done.

Consider now the general case.

Claim 1. We can restrict to the case $V=\mathbb{K}^{k}$.

By Corollary 3.8, it suffices to prove that for every $a \in \Delta_{f}$ there exists a neighbourhood $U_{a}$ of $a$ such that $f\left(U_{a} \cap \Delta_{f}\right)$ is meager. Fix $a \in \Delta_{f}$. Using the Implicit Function Theorem, it is easy to check that there is a neighbourhood $U_{a}$ of $a$ and a definable diffeomorphism $\Phi: \mathbb{K}^{k} \rightarrow V \cap U_{a}$ such that $H \circ \Phi \equiv 0$ and each $F_{i} \circ \Phi$ belongs to a Noetherian differential ring $M^{\prime} \subseteq \mathcal{C}^{\infty}\left(\mathbb{K}^{k}, \mathbb{K}\right)$ (see [21] for the details). Hence Claim 11 is proved and we may assume that $f: \mathbb{K}^{k} \rightarrow \mathbb{K}^{m}$, and $f \in M \subseteq \mathcal{C}^{\infty}\left(\mathbb{K}^{k}, \mathbb{K}\right)$.

Let $X_{0}:=\left\{a \in \Delta_{f}: D f(a) \neq 0\right\}$, where $D f$ is the Jacobian matrix of $f$. We first prove that $f\left(X_{0}\right)$ is meager.

Again by Corollary 3.8 , it suffices to prove that for every $a \in X_{0}$ there exists a neighbourhood $U_{a}$ of $a$ such that $f\left(U_{a} \cap X_{0}\right)$ is meager.

Fix $a \in X_{0}$.

Claim 2. We may assume that $f(x)=\left(x_{1}, f_{2}(x), \ldots, f_{m}(x)\right)$.

In fact, since $D f(a) \neq 0$, we can assume that $\partial f_{1}(a) / \partial x_{1} \neq 0$ and $a=0$.

Consider definable neighbourhoods $O$ and $\tilde{O} \subset \mathbb{K}^{k}$ of 0 , where the following map is a diffeomorphism:

$$
G: O \rightarrow \tilde{O}, \quad x \mapsto\left(f_{1}(x), x_{2}, \ldots, x_{k}\right) .
$$

Let $\Delta$ be the determinant of the Jacobian of $G$ and let $\hat{M}:=\left\{g \circ G^{-1}\right.$ : $g \in M\} \subset \mathcal{C}^{\infty}(\tilde{O}, \mathbb{K})$; then the ring $\tilde{M}:=\hat{M}\left[\Delta^{-1}\right]$ is clearly Noetherian and differentially closed; define $\tilde{f}:=f \circ G^{-1} \in \tilde{M}$. Since $G$ is a diffeomorphism, it is enough to prove the statement for $\tilde{M}$ and $\tilde{f}$, and Claim 2 is proved.

For every $t \in \mathbb{K}$, consider the Noetherian differential ring

$$
N_{t}:=\left\{g_{t}:=g\left(t, x_{2}, \ldots, x_{k}\right): g \in M\right\} \subset \mathcal{C}^{\infty}\left(\tilde{O} \cap \mathbb{K}^{k-1}, \mathbb{K}\right) .
$$

Let $f_{t}: \mathbb{K}^{k-1} \rightarrow \mathbb{K}^{m-1}$ be the map $\left(\left(f_{2}\right)_{t}, \ldots,\left(f_{m}\right)_{t}\right)$. By inductive hypothesis, the set $\Sigma_{f_{t}}$ is meager in $\mathbb{K}^{m-1}$. Moreover, $f\left(X_{0} \cap \tilde{O}\right) \cap\left(\{t\} \times \mathbb{K}^{m-1}\right) \subseteq$ $\{t\} \times \Sigma_{f_{t}}$. Hence $f\left(X_{0} \cap \tilde{O}\right) \subseteq D:=\left\{(t, y) \in \mathbb{K} \times \mathbb{K}^{k-1}: y \in \Sigma_{f_{t}}\right\}$. By what we have just observed, $T(D):=\left\{t \in \mathbb{K}: D_{t}\right.$ is meager $\}$ is residual, because $D_{t}=\Sigma_{f_{t}}$, hence by Proposition 5.4 . $D$ is meager. It follows by Corollary 3.8 that $f\left(X_{0}\right)$ is meager. 
Now, let $a \in \Delta_{f}$ be such that $\operatorname{Df}(a)=0$, and let $P$ be the least natural number such that there exists $i \leq m$ and a derivative $\theta$ of order $P$ such that if $g_{\theta}:=\theta f_{i}$, then $g_{\theta}(a)=0$ and $D g_{\theta}(a) \neq 0$. Such a $P$ exists by Proposition 6.6. Let $W_{\theta}:=V^{\mathrm{reg}}\left(g_{\theta}\right) \subset \mathbb{K}^{k}$ (notice that the inclusion is strict, hence $\operatorname{dim} W_{\theta}<k$ ). Then there is a definable open neighbourhood $O$ of $a$ such that

$$
\Delta_{f} \cap O \subseteq \bigcup_{\operatorname{ord}(\theta) \leq P} W_{\theta} .
$$

Hence it is enough to prove that $f\left(\Delta_{f} \cap W_{\theta}\right)$ is meager. Let $h_{\theta}:=f\left\lceil W_{\theta}\right.$. By inductive hypothesis, $\Sigma_{h_{\theta}}$ is meager. Note that if $x \in W_{\theta}$ is a singular point for $f$, then $x$ is also a singular point for $h_{\theta}$; that is, $\Delta_{f} \cap W_{\theta} \subseteq \Delta_{h_{\theta}}$, and we are done.

Corollary 6.9. Let $F \in M^{k}$ and $G \in M$. Define $X:=V^{\mathrm{reg}}(F) \subseteq U$, and, for every $\bar{a} \in \mathbb{K}^{n}, g_{\bar{a}}: X \rightarrow \mathbb{K}$ as $g_{\bar{a}}(\bar{x}):=G(\bar{x})+\sum a_{i} x_{i}$. Then the set $A=\left\{\left(a_{1}, \ldots, a_{n}\right) \in \mathbb{K}^{n}: g_{\bar{a}}\right.$ is not a Morse function $\left(^{3}\right)$ on $\left.X\right\}$ is meager.

Proof. We proceed as in [12].

Claim 1. The corollary is true if $k=0$, i.e. if $X=U$.

In fact, $\bar{a} \in A$ iff $-\bar{a}$ is a critical value of $\nabla G$, and we can apply Theorem 6.8 .

By the Implicit Function Theorem, around every point $p \in X$ there exists an open definable neighbourhood $U_{p}$ such that the restriction of some $n-k$ of the coordinate functions on $\mathbb{K}^{n}$ (say, the first $n-k$ ) constitute a coordinate system in $U_{p}$; let $V_{p}:=\Pi_{n-k}^{n}\left(U_{p}\right)$ and $\phi_{p}: V_{p} \rightarrow U_{p}$ be the inverse map of $\Pi_{n-k}^{n} \mid U_{p}$. Let $\tilde{M}$ be the ring of functions on $V_{p}$ of the form $h \circ \phi$, where $h \in M$ : notice that $\tilde{M}$ is contained in some Noetherian differential ring $M_{p}$ (see [21]). Let $A_{p}=\left\{\left(a_{1}, \ldots, a_{n}\right) \in \mathbb{K}^{n}: g_{\bar{a}}\right.$ is not a Morse function on $\left.V_{p}\right\}$. Proceeding as in [12], using Proposition 5.4 instead of Fubini's theorem, and Claim 1 applied to functions in the ring $M_{p}$, we see that $A_{p}$ is meager for every $p \in X$. Since $A=\bigcup_{p \in X} A_{p}$, Corollary 3.9 implies that $A$ is meager.

7. Pfaffian functions. Let $\mathbb{K}$ be a definably complete Baire structure.

Definition 7.1. Let $f_{1}, \ldots, f_{s}: \mathbb{K}^{n} \rightarrow \mathbb{K}$ be definable and $\mathcal{C}^{1}$. We say that $\left(f_{1}, \ldots, f_{s}\right)$ is a Pfaffian chain (in $\mathbb{K}$ ) of length $s$ if $\partial f_{i} / \partial x_{j} \in$ $\mathbb{K}\left[\bar{x}, f_{1}, \ldots, f_{i}\right]$ for $i=1, \ldots, s$ and $j=1, \ldots, n$. A definable map $F=$ $\left(F_{1}, \ldots, F_{m}\right): \mathbb{K}^{n} \rightarrow \mathbb{K}^{m}$ is Pfaffian (in $\left.\mathbb{K}\right)$ if $F_{1}, \ldots, F_{m} \in \mathbb{K}\left[\bar{x}, f_{1}, \ldots, f_{s}\right]$ for some Pfaffian chain $\left(f_{1}, \ldots, f_{s}\right)$.

$\left({ }^{3}\right)$ A definable $\mathcal{C}^{2}$ function $f$, from a $\mathcal{C}^{2} \mathbb{K}$-manifold to $\mathbb{K}$, is a Morse function if, as in the classical definition, every singular point of $f$ is nondegenerate. 
Consider polynomials $p_{i j} \in \mathbb{K}\left[\bar{x}, y_{1}, \ldots, y_{i}\right], q_{k} \in \mathbb{K}\left[\bar{x}, y_{1}, \ldots, y_{s}\right]$ such that

$$
\begin{array}{rlrl}
\frac{\partial f_{i}}{\partial x_{j}}(\bar{x}) & =p_{i j}\left(\bar{x}, f_{1}(\bar{x}), \ldots, f_{i}(\bar{x})\right), & & i \leq s, j \leq n, \\
F_{k}(\bar{x}) & =q_{k}\left(\bar{x}, f_{1}(\bar{x}), \ldots, f_{s}(\bar{x})\right), \quad k \leq m .
\end{array}
$$

The complexity of $F$ is the sequence of integers $\left(n, m, s, \operatorname{deg} q_{k}, \operatorname{deg} p_{i j}\right.$ : $i \leq s, j \leq n, k \leq m)$.

We prove the following version of Khovanskii's Theorems (see 14, Theorems 1 and 2]).

THEOREM 7.2.

(1) Suppose $F: \mathbb{K}^{n} \rightarrow \mathbb{K}^{n}$ is Pfaffian. Then the number of regular zeros of $F$ is finite and can be bounded by a function of the complexity of $F$.

(2) Suppose $F: \mathbb{K}^{n} \rightarrow \mathbb{K}^{m}$ is Pfaffian. Then the number of definably connected components of $F^{-1}(0)$ is finite and can be bounded by a function of the complexity of $F$.

The fact that the bounds in the above theorem depend only on the complexity implies, in particular, that they do not depend on the coefficients of the polynomials in the Pfaffian chain, or on other parameters in the definition of $F$. Moreover, the reader can verify that the explicit bounds given in [14 continue to work in this context.

7.1. Proof of Theorem 7.2. We will follow the outline of [16]; the results that we do not prove here are easy modifications of the ones in [16].

We prove the first statement by induction on the length $s$ of the Pfaffian chain; the second one will follow from the first. If $s=0$ then $F$ is a polynomial map, and the bound in the first and the second statement is given by [2, Proposition 11.5.4].

Let $s>0$, and suppose that, for all Pfaffian chains of length $\leq s-1$, the first statement of Theorem 7.2 holds true.

In the following proof, notice that the various reductions might increase the complexity of the Pfaffian functions involved; however, the length will only stay the same or decrease.

Let $F: \mathbb{K}^{n} \rightarrow \mathbb{K}^{n}$ be Pfaffian with respect to a Pfaffian chain $\bar{f}=$ $\left(f_{1}, \ldots, f_{s}\right)$, with $F=\left(F_{1}, \ldots, F_{n}\right)$ and $F_{i}(\bar{x})=q_{i}\left(\bar{x}, f_{1}(\bar{x}), \ldots, f_{s}(\bar{x})\right)$.

Define $H: \mathbb{K}^{n+1} \rightarrow \mathbb{K}^{n}$ and $G: \mathbb{K}^{n+1} \rightarrow \mathbb{K}$ as follows:

$$
\begin{aligned}
H_{i}(\bar{x}, y) & :=q_{i}\left(\bar{x}, f_{1}(\bar{x}), \ldots, f_{s-1}(\bar{x}), y\right), \quad i=1, \ldots, n, \\
G(\bar{x}, y) & :=y-f_{s}(\bar{x}) .
\end{aligned}
$$

Remark 7.3. $H$ and $G$ are Pfaffian maps. Moreover,

(1) $H$ has length $s-1$ and $G$ has length $s$. 
(2) The complexities of $G$ and $H$ depend only on the complexity of $F$.

(3) $V(G)=V^{\mathrm{reg}}(G)$.

(4) If $\bar{a} \in V^{\mathrm{reg}}(F) \subseteq \mathbb{K}^{n}$, then there exists $b \in \mathbb{K}$ such that $(\bar{a}, b) \in$ $V^{\mathrm{reg}}(H, G) \subseteq \mathbb{K}^{n+1}$.

Hence it is enough to bound the cardinality of $V^{\mathrm{reg}}(H, G)$.

Definition 7.4. A definable continuous function $f: \mathbb{K}^{d} \rightarrow \mathbb{K}^{d^{\prime}}$ is proper if the preimage of every d-compact set is d-compact. Notice that $f$ is proper iff $\lim _{|\bar{x}| \rightarrow \infty}|f(\bar{x})|=+\infty$.

Lemma 7.5. We may assume that $H$ is proper.

Proof. In [16, bottom of p. 183] it is shown how to reduce to the case when $q_{n}$ does not depend on $f_{1}, \ldots, f_{s}$, and $\lim _{|\bar{x}| \rightarrow \infty}\left|q_{n}(\bar{x})\right|=+\infty$; in this case, $H_{n}(\bar{x}, y)=q_{n}(\bar{x})$, and thus $H$ is proper.

Lemma 7.6. We may assume that $V(H)=V^{\mathrm{reg}}(H)$ and $V(H, G)=$ $V^{\mathrm{reg}}(H, G)$.

Proof. Suppose this is not the case. For every $\bar{b} \in \mathbb{K}^{n}$, we consider the Pfaffian proper map $H_{\bar{b}}:=H-\bar{b}$. Note that $H_{\bar{b}}$ has length $s-1$ and the same complexity as $H$.

Let $B$ be the set of all $\bar{b} \in \mathbb{K}^{n}$ such that $V\left(H_{\bar{b}}\right)=V^{\text {reg }}\left(H_{\bar{b}}\right)$ and $V\left(H_{\bar{b}}, G\right)=V^{\text {reg }}\left(H_{\bar{b}}, G\right)$. By Theorem 6.8, $B$ is a residual subset of $\mathbb{K}^{n}$. Suppose we did prove that, for every $\bar{b} \in B,\left|V^{\text {reg }}\left(H_{\bar{b}}, G\right)\right| \leq N$. The set of all $\bar{a} \in \mathbb{K}^{n}$ such that $\left|V^{\mathrm{reg}}\left(H_{\bar{a}}, G\right)\right| \geq N+1$ is open (by the Implicit Function Theorem, applied to $H$ restricted to the manifold $V(G)=V^{\mathrm{reg}}(G)$ ) and disjoint from $B$, and therefore empty.

We have thus reduced our problem to the following situation: $\Gamma:=$ $V^{\text {reg }}(H)=V(H) \subseteq \mathbb{K}^{n+1}$ is a smooth d-compact Pfaffian curve of length $s-1$ and $G: \mathbb{K}^{n+1} \rightarrow \mathbb{K}$ is a Pfaffian map of length $s$ such that $g:=G\lceil\Gamma$ has only regular zeros. Notice that we have never changed the definition of $G=y-f_{s}(\bar{x})$ in the above reductions. We need to bound the number of zeros of $g$ (which will be done in Lemma 7.14).

Definition 7.7. Given a $\mathcal{C}^{1}$ function $f: \mathbb{K}^{d} \rightarrow \mathbb{K}^{d}$, let $J(f): \mathbb{K}^{d} \rightarrow \mathbb{K}$ be the determinant of the Jacobian matrix of $f$.

Definition 7.8. An arc of a nonsingular curve $\Gamma$ is the image of a definable differentiable function $\phi: I \rightarrow \Gamma$ such that $I \subseteq \mathbb{K}$ is an interval and $\phi^{\prime}(t)$ is nonzero for all $t \in I$. The function $\phi$ is called a parametrization of the arc. When no confusion is possible we use the word "arc" both for $\phi$ and its image.

In [16, pp. 184-186], the proof continues as follows: since $\Gamma$ is a compact $\mathcal{C}^{1}$ curve of dimension 1 , it has a finite number of connected components 
$\Gamma_{1}, \ldots, \Gamma_{r}$, each of which is diffeomorphic to the unit circle $S^{1}$. Moreover, $g$ has a finite number of zeros (because $V(g)$ is discrete and compact). Hence, for $i=1, \ldots, r$, there is a parametrization $\phi: \mathbb{R} \rightarrow \Gamma_{i}$, and using such parametrization we can speak about consecutive zeros of $g$ (Definition 7.10). Set $\tilde{g}:=g \circ \phi: \mathbb{R} \rightarrow \mathbb{R}$; since all the zeros of $g$ (and hence all zeros of $\tilde{g}$ ) are regular, the derivative $\tilde{g}^{\prime}$ changes sign at two consecutive zeros of $\tilde{g}$ (Rolle's Theorem). Hence, there is a zero $z$ of $\tilde{g}^{\prime}$ between each pair of consecutive zeros of $\tilde{g}$, and therefore $\phi(z)$ is a zero of $L:=(H, J(H, G))$; thus, $|V(g)| \leq$ $|V(L)|$. However, the length of $L$ is still $s$, thus we cannot use the inductive hypothesis to bound $V(L)$ (even assuming that 0 is a regular value of $L$ ).

Khovanskii's idea was to define another function $\hat{J}: \mathbb{K}^{n+1} \rightarrow \mathbb{K}$ of length $s-1$ such that $\hat{J}$ and $J(H, G)$ coincide on $V(G)$ (actually, it is enough that $\hat{J}$ and $J(H, G)$ coincide on $V(H, G))$. Let $\hat{\jmath}:=\hat{J}\lceil\Gamma$; notice that $\hat{\jmath}$ changes sign between two consecutive zeros of $g$, because $\hat{\jmath}$ coincides with $J(H, G)$ on $V(g)$; thus, $|V(\hat{\jmath})| \geq|V(g)|$. The last trick is to show that if $(*)\left|V^{\text {reg }}\left(\hat{\jmath}_{t}\right)\right| \leq N$ for every $t \in \mathbb{K}$, then $|V(g)| \leq N$, where $\hat{\jmath}_{t}:=\hat{\jmath}-t$. Finally, we can apply the inductive hypothesis to $\hat{\jmath}_{t}$ for every $t$, and obtain a bound $N$ satisfying $(*)$.

To generalize to our situation, the main difficulty lies in proving the existence of a parametrization for $\Gamma$.

Proposition 7.9. $\Gamma$ is the union of finitely many arcs.

Proof. Let $0 \neq \bar{a}=\left(a_{1}, \ldots, a_{n}\right) \in \mathbb{K}^{n}$ be such that the function $u: \Gamma \rightarrow$ $\mathbb{K}$ given by $\bar{x} \mapsto \bar{a} \cdot \bar{x}$ is Morse (such an $\bar{a}$ exists by Corollary 6.9).

Claim 1. There exists an effective finite bound $N$ on the cardinality of the set of critical points of $u$, in terms of the complexity of $H$.

In fact, each critical point of $u$ is a regular point of $(H, J(H, U))$, where $U(\bar{x}):=\bar{a} \cdot \bar{x}$, and we can apply the inductive hypothesis to the latter map.

For every $t \in \mathbb{K}$, let $\Gamma_{t}:=u^{-1}(t)$; notice that, since $u$ is Morse, $\Gamma_{t}$ is a discrete set.

Claim 2. For every $t \in \mathbb{K},\left|\Gamma_{t}\right| \leq 2 N$.

In fact, let $c_{1}<\cdots<c_{m}$ be the critical values of $u$. For every $t \in \mathbb{K}$, let $\Delta_{t}$ be the set of singular points of $u$ in $\Gamma_{t}$. Fix $t \in\left[c_{1}, c_{m}\right]$. Since $u$ is a Morse function, $\Gamma_{t}$ and $\Delta_{t}$ are discrete and d-compact.

Using the Implicit Function Theorem and the fact that $u$ is Morse, we see that for every $x \in \Gamma_{t}$ there exists a definable neighbourhood $V_{x}$ of $x$ and $\varepsilon_{x}>0$ such that:

$\circ$ if $x$ is a regular point of $u$, then $\left|V_{x} \cap \Gamma_{t^{\prime}}\right|=1$ for every $t^{\prime} \in B\left(x ; \varepsilon_{x}\right)$;

$\circ$ if $x$ is a singular point of $u$, then $\left|V_{x} \cap \Gamma_{t^{\prime}}\right| \leq 2$ for every $t^{\prime} \in B\left(x ; \varepsilon_{x}\right)$. 
Since $\Gamma_{t}$ is discrete and d-compact, the sets $V_{x}$ can be chosen to be pairwise disjoint and we can choose $\varepsilon$ independently of $x$. By possibly reducing $\varepsilon$, we can prove the following:

o If $t$ is a regular value of $f$, then $\left|\Gamma_{t}\right|=\left|\Gamma_{t^{\prime}}\right|$ for every $t^{\prime} \in B(t ; \varepsilon)$.

- If $t$ is a singular value of $f$, then ||$\Gamma_{t}|-| \Gamma_{t^{\prime}}|| \leq\left|\Delta_{t}\right|$ for every $t^{\prime} \in$ $B(t ; \varepsilon)$.

We can conclude that for every $i=1, \ldots, m-1$, and every $t, t^{\prime} \in\left(c_{i}, c_{i+1}\right)$, either both $\Gamma_{t}$ and $\Gamma_{t^{\prime}}$ are infinite, or $\left|\Gamma_{t}\right|=\left|\Gamma_{t^{\prime}}\right|$. Hence, $\left|\Gamma_{c_{1}}\right|=\left|\Delta_{c_{1}}\right| ;\left|\Gamma_{t}\right| \leq$ $2\left|\Delta_{c_{1}}\right|$ for all $t \in\left(c_{1}, c_{2}\right) ;\left|\Gamma_{c_{2}}\right| \leq 2\left|\Delta_{c_{1}}\right|+\left|\Delta_{c_{2}}\right| ; \ldots$

This completes the proof of the claim.

Claim 3. Let $n, N \in \mathbb{N}$. There is a subset $D(n, N) \subset \mathbb{Z}^{n}$ of cardinality $n N^{2}+1$ such that for every $N$-tuple of distinct points $p_{1}, \ldots, p_{N} \in \mathbb{K}^{n}$ there is $Z \in D(n, N)$ such that all the scalar products $Z \cdot p_{1}, \ldots, Z \cdot p_{N}$ are distinct elements of $\mathbb{K}$.

For a proof, see for example [22, Lemma 6.1].

Claim 4. Let $Z \in \mathbb{K}^{n}$. The set $S_{Z, m}=\left\{t \in \mathbb{K}:\left|Z \cdot \Gamma_{t}\right| \geq m\right\}$ is a finite union of open intervals and points.

In fact, the set $S_{Z, m}$ is the projection onto the $t$-axis of the zero-set of the Pfaffian map (of length $s-1) R(\bar{x}, \bar{w}, t)$ which sends $\left(\bar{x}_{1}, \ldots, \bar{x}_{m}\right.$, $\left.w_{1,2}, \ldots, w_{m-1, m}, t\right)$ to $\left(H\left(\bar{x}_{1}\right), \ldots, H\left(\bar{x}_{m}\right), u\left(\bar{x}_{1}\right)-t, \ldots, u\left(\bar{x}_{m}\right)-t, \ldots,\left(Z \cdot \bar{x}_{i}-Z \cdot \bar{x}_{j}\right) w_{i, j}-1, \ldots\right)$.

By [21, Theorem 45], $V(R)$ has finitely many definably connected components, hence so does its projection $S_{Z, m}$.

Finally, we can prove the proposition. Since $\Gamma$ is a nonsingular curve, it is a union of arcs. We must show that finitely many arcs suffice. By Corollary 6.9, there is a linear function $U: \mathbb{K}^{n+1} \rightarrow \mathbb{K}$ whose restriction $u$ to $\Gamma$ is a Morse function. By Claim 1, $u$ has a finite number of critical points, and by Claim 2 we can find $N \in \mathbb{N}$ such that each fibre $\Gamma_{t}$ has cardinality at most $N$. Fix $D(n+1, N) \subset \mathbb{Z}^{n+1}$ as in Claim 3. By Claim 4 for each $Z \in D(n+1, N)$ and $m \in \mathbb{N}$, the set $S_{Z, m}=\left\{t \in \mathbb{K}:\left|Z \cdot \Gamma_{t}\right| \geq m\right\}$ is a finite union of open intervals and points. Since $S_{Z, m}$ is empty for $m>N$, the Boolean algebra generated by the sets $S_{Z, m}$ is finite. Its atoms give a partition $\mathcal{D}$ of $\mathbb{K}$ into a finite union of open intervals and points. If $I \in \mathcal{D}$, then $\left|Z \cdot \Gamma_{t_{1}}\right|=\left|Z \cdot \Gamma_{t_{2}}\right|$ for all $t_{1}, t_{2} \in I$ and all $Z \in D(n+1, N)$. By Claim 3 it then follows that there is $Z_{I} \in D(n+1, N)$ such that $\left|Z_{I} \cdot \Gamma_{t}\right|=\left|\Gamma_{t}\right|$ for all $t \in I$. So in particular there is $k_{I} \in \mathbb{N}$ such that $\left|\Gamma_{t}\right|=k_{I}$ for all $t \in I$. By Claim 1, $u$ has finitely many critical points, so we can assume, refining the partition, that no interval $I \in \mathcal{D}$ contains critical values of $u$. We order 
the finite set $Z_{I} \cdot \Gamma_{t}$ by the order it inherits as a subset of $\mathbb{K}$. For $i \leq k_{I}$ let $\phi_{I, i}(t) \in \Gamma_{t}$ be such that $Z_{I} \cdot \phi_{I, i}(t)$ is the $i$ th element of $Z_{I} \cdot \Gamma_{t}$.

We claim that $\phi_{I, i}$ is continuous. This is clear if $I$ is a point, so suppose it is an interval. Then by our assumptions $I$ does not contain critical points of $u$. Fix $t \in I$. To prove the continuity of $\phi_{I, i}$ at $t$, let $\Gamma_{t}=\left\{\bar{x}_{1}, \ldots, \bar{x}_{k_{I}}\right\}$, where $\bar{x}_{i}=\phi_{I, i}(t)$. Since $\bar{x}_{i}$ is not a critical point of $u$, we can choose an open neighbourhood $U_{i}$ of $\bar{x}_{i}$ which is mapped by $u$ diffeomorphically onto an open interval $(a, b) \subseteq I$ containing $t$. By choosing the $U_{i}$ small enough we can assume that $Z_{I} \cdot U_{1}, \ldots, Z_{I} \cdot U_{k_{I}}$ are disjoint open intervals of $\mathbb{K}$ and that $u\left(U_{i}\right)=(a, b)$ for every $i$. It then follows that $\phi_{I, i}$ maps $(a, b)$ into $U_{i}$ and it coincides with the inverse of the diffeomorphism $u\left\lceil U_{i}\right.$. Thus $\phi_{I, i}$ is continuous.

The curve $\Gamma$ is the union of finitely many arcs parametrized by the functions $\phi_{I, i}$, with $I$ an interval of $\mathcal{D}$, together with finitely many points (the images of the functions $\phi_{I, i}$ when the set $I \in \mathcal{D}$ is just a point). To complete the proof it suffices to observe that since $\Gamma$ is a nonsingular curve, each of these finitely many points is contained in some arc.

Definition 7.10. Let $\xi_{H}$ be the unique vector field on $\mathbb{K}^{n+1}$ such that for every smooth definable function $g: \mathbb{K}^{n+1} \rightarrow \mathbb{K}$ we have $\xi_{H}(\bar{x}) \cdot \nabla g(\bar{x})=$ $J(H, g)(\bar{x})$. Note that $\xi_{H}$ is tangent to $\Gamma$ and is never zero on $\Gamma$. We say that the arc $\phi: I \rightarrow \Gamma$ is orientation preserving if $\phi^{\prime}(t) \cdot \xi_{H}(\phi(t))>0$ for every $t \in I$. Note that if $\phi:(a, b) \rightarrow \Gamma$ is not orientation preserving, then its reverse arc $-\phi(t):=\phi(b-t+a)$ is orientation preserving.

We say that two points $\bar{x}, \bar{y} \in V(g)$ are consecutive if there are an orientation preserving $\operatorname{arc} \phi: I \rightarrow \Gamma=V(H)$ and $t_{1}<t_{2}$ in $I$ such that $\bar{x}=\phi\left(t_{1}\right)$, $\bar{y}=\phi\left(t_{2}\right)$ and $\phi(t) \notin V(g)$ for every $t \in\left(t_{1}, t_{2}\right)$.

The proof of the following lemma is elementary.

Lemma 7.11. Let $\bar{x}, \bar{y}$ be consecutive points in $V(g)$. Then $J(H, G)$ assumes opposite signs at $\bar{x}, \bar{y}$. So in particular $\bar{x} \neq \bar{y}$.

Lemma 7.12. For each $\bar{x} \in V(g)$, there is $\bar{y} \in V(g)$ such that $\bar{x}, \bar{y}$ are consecutive.

Proof. In the case when $\mathbb{K}$ expands $\mathbb{R}$, one would use the fact that $V(g)$ is finite; but we do not know it yet in our situation. However, to prove the conclusion it suffices to notice that $V(g)$ is discrete and d-compact.

Lemma 7.13. There is a Pfaffian function $\hat{J}: \mathbb{K}^{n+1} \rightarrow \mathbb{K}$ of length $s-1$ which coincides with $J(H, G)$ on $V(G)$.

As we said before, $\hat{\jmath}:=\hat{J}\lceil\Gamma$ assumes opposite signs at two consecutive points $\bar{x}, \bar{y}$ of $V(g)$. Hence, reasoning as in [16, Lemma 3.1], we can prove the following lemma. 
Lemma 7.14. $V(g)$ is finite, and we can compute a bound on its cardinality in terms of the complexity of $(H, G)$.

Proof. Let $\varepsilon$ be the minimum of the absolute value of $\hat{\jmath}$ on the closed and bounded set $V(g)$. Then $\hat{\jmath}$ assumes every value between $-\varepsilon$ and $+\varepsilon$ between any two consecutive points $\bar{x}, \bar{y}$ of $V(g)$. By Theorem 6.8, $\hat{\jmath}$ has a regular value $t \in(-\varepsilon,+\varepsilon)$. Since $\hat{J}$ has length $\leq s-1$, using the inductive hypothesis we can compute a finite bound on the cardinality of $\hat{\jmath}^{-1}(t)$. This is also a bound on $V(g)$ since we can associate injectively to each $\bar{x} \in V(g)$ a point of $\hat{\jmath}^{-1}(t)$ lying in the arc between $\bar{x}$ and the point consecutive to $\bar{x}$ (which exists by Lemma 7.12).

Combining all the lemmas, we obtain a proof of the first statement of Theorem 7.2 .

We now prove the second statement. Let $F: \mathbb{K}^{n} \rightarrow \mathbb{K}^{m}$ be Pfaffian with respect to a Pfaffian chain $\left(f_{1}, \ldots, f_{s}\right)$. We need some preliminary results.

Definition 7.15. Let Cofin $(\mathbb{K})$ be the cofinality of $\mathbb{K}$. A sequence is a $\operatorname{map} x: \operatorname{Cofin}(\mathbb{K}) \rightarrow \mathbb{K}^{m}$. If $\left(x_{k}\right)_{k<\operatorname{Cofin}(\mathbb{K})}$ is a sequence, we say that $x_{k} \rightarrow l$ if for every neighbourhood $V$ of $l$ there exists $\mu<\operatorname{Cofin}(\mathbb{K})$ such that $x_{k} \in V$ for every $k>\mu$. We call $\left(x_{k}\right)_{k<\operatorname{Cofin}(\mathbb{K})}$ infinitesimal if $x_{k} \rightarrow 0$.

The following lemma is a modification of an observation by Alessio Martini.

Lemma 7.16. Let $F: \mathbb{K}^{n} \rightarrow \mathbb{K}$ be definable, continuous, proper and nonnegative, and $M \in \mathbb{N}$. Suppose there is an infinitesimal nonnegative sequence $\left(\varepsilon_{k}\right)_{k<\operatorname{Cofin}(\mathbb{K})}$ such that for every $k<\operatorname{Cofin}(\mathbb{K}), F^{-1}\left(\varepsilon_{k}\right)$ has fewer than $M$ definably connected components. Then $F^{-1}(0)$ has fewer than $M$ definably connected components.

Proof. Since $F$ is proper, $F^{-1}(0)$ and $F^{-1}\left(\varepsilon_{k}\right)$ are d-compact. Assume, for contradiction, that there exists a partition $\left\{C_{0}, \ldots, C_{M-1}\right\}$ of $F^{-1}(0)$ into nonempty definable clopen subsets. Let

$$
\begin{gathered}
\delta:=\frac{1}{3} \min _{i \neq j} d\left(C_{i}, C_{j}\right), \quad W:=\left\{\bar{x} \in \mathbb{K}^{n}: d\left(\bar{x}, F^{-1}(0)\right)<\delta\right\}, \\
B_{i}:=\left\{\bar{x} \in \mathbb{K}^{n}: d\left(\bar{x}, C_{i}\right)<\delta\right\}, \quad J_{i}:=F\left(B_{i}\right) .
\end{gathered}
$$

Note that $\delta>0$, the $B_{i}$ s are open (in $K^{n}$ ) and disjoint, $B_{i} \cap F^{-1}(0)=C_{i}$, and $W=\bigcup_{i} B_{i}$. We note that each $J_{i}$ is definably connected. Indeed, assume, say, $i=0$. Let $\varepsilon \in J_{0}$, and let $\bar{y} \in B_{0}$ be such that $F(\bar{y})=\varepsilon$. Let $\bar{x} \in C_{0}$ be such that $d(\bar{x}, \bar{y})<\delta$. Note that the segment $[\bar{x}, \bar{y}]$ is contained in $B_{0}$. Since $[\bar{x}, \bar{y}]$ is definably connected, $F([\bar{x}, \bar{y}])$ is also definably connected, and therefore $\varepsilon$ is in the same definably connected component of $J_{0}$ as 0 ; since this is true for every $\varepsilon \in J_{0}, J_{0}$ is definably connected. 
Let $\theta_{i}:=\sup J_{i}$ and $\eta_{1}:=\min _{i} \theta_{i}$. We claim that there exists $\eta_{2}>0$ such that $F^{-1}\left(\left[0, \eta_{2}\right)\right) \subseteq W$. Let $D:=F^{-1}([0,1]) \backslash W$. Note that $D$ is d-compact, because $F$ is proper. If $D=\emptyset$, we can define $\eta_{2}=1$. Otherwise, $F(D)$ is d-compact and nonempty. Let $\eta_{2}:=\min D$. Since $F^{-1}(0) \cap D=\emptyset$, we have $\eta_{2}>0$. Let $F(\bar{x})<\eta_{2}$. Then $\bar{x} \notin D$, and therefore $\bar{x} \in W$.

Define $\eta=\min \left(\eta_{1}, \eta_{2}\right)$. Therefore, for every $\varepsilon<\eta$, we have

$$
F^{-1}(\varepsilon) \subseteq \bigcup_{i} B_{i} \text { and } \varepsilon \in \bigcap_{i} F\left(B_{i}\right) .
$$

Let $k<\operatorname{Cofin}(\mathbb{K})$ be such that $\varepsilon_{k}<\eta$. Since $F^{-1}\left(\varepsilon_{k}\right)$ has at most $M$ definably connected components, we deduce that $F^{-1}\left(\varepsilon_{k}\right) \cap B_{i}=\emptyset$ for at least one $i$. However, this contradicts $\varepsilon_{k} \in \bigcap_{i} F\left(B_{i}\right)$.

We turn to the proof of the second statement of Theorem 7.2 .

Lemma 7.17. We may assume that $F$ is proper, $m=1$ and $F \geq 0$.

Lemma 7.18. We may assume that 0 is a regular value for $F$.

Proof. Consider the function $F_{\varepsilon}:=F-\varepsilon$ for $0<\varepsilon \in \mathbb{K}$. It follows from Theorem 6.8 that the set of critical values of $F$ is meager, hence we can find an infinitesimal sequence $\left(\varepsilon_{n}\right)_{n<\text { Cofin( }(\mathbb{K})}$ such that $\varepsilon_{n}$ is a regular value for $F$. If we find a bound which works for every $F_{\varepsilon_{n}}$, then by Lemma 7.16 , the same bound will work for $F$.

By Corollary 6.9, there exists a nonzero $\bar{a} \in \mathbb{K}^{n}$ such that $u_{\bar{a}}(\bar{x}):=\bar{a} \cdot \bar{x}$ is a Morse function on $V(F)$. Once these lemmas are established, note that every nonempty clopen definable subset $C$ of $V(F)$ is d-compact, and hence the function $u_{\bar{a}}$ has at least one critical point on $C$; it follows by a standard argument that the number of definably connected components of $V(F)$ is finite, and is bounded by the number of critical points of $u_{\bar{a}}$ on $V(F)$, if the latter is also finite.

Choose an orthogonal basis $e_{1}, \ldots, e_{n}$ of the orthogonal space to $\bar{a}$, and write $\left(y_{1}, \ldots, y_{n-1}, y_{n}\right)$ for the coordinates in the basis $\left(e_{1}, \ldots, e_{n}, \bar{a}\right)$. We can then proceed as in [16, pp. 188-189]: a calculation shows that the critical points of $u_{\bar{a}}$ on $V(F)$ are regular zeros of the map $\tilde{F}:=\left(F, \partial F / \partial y_{1}, \ldots\right.$ $\left.\ldots, \partial F / \partial y_{n-1}\right)$. Since each $\partial F / \partial y_{i}$ is a linear combination of $\partial F / \partial x_{1}, \ldots$ $\partial F / \partial x_{n}$, a bound on $\left|V^{\mathrm{reg}}(\tilde{F})\right|$ is given by the first statement in Theorem 7.2 . This concludes the proof.

8. Conclusion. Now we are ready to give necessary and sufficient conditions for a definably complete Baire expansion of a field by $\mathcal{C}^{\infty}$ functions to be o-minimal:

THEOREM 8.1. Let $\mathbb{K}$ be a definably complete Baire expansion of a field by a family of $\mathcal{C}^{\infty}$ functions. Then $\mathbb{K}$ is o-minimal if and only if every quan- 
tifier free $\mathbb{K}$-definable set has finitely many definably connected components (uniformly in the parameters).

This result, together with Theorem 7.2 , gives us immediately the following corollary.

COROllary 8.2. Let $\mathbb{K}$ be a definably complete Baire expansion of a field by a family of Pfaffian functions. Then $\mathbb{K}$ is o-minimal.

REMARK 8.3. We recall that Tarski's question about the decidability of the theory of the real exponential field is still open. The best result so far in this direction is in [15], where the authors give a candidate for an axiomatization, which is actually complete if Schanuel's Conjecture is true. It follows from Corollary 8.2 that if $L$ is the language of ordered rings with an extra unary function symbol $f$, then the $L$-theory axiomatized by the scheme of definably complete Baire structure together with an axiom stating that $f^{\prime}=f$ and $f(0)=1$, is a recursive and o-minimal subtheory of the theory of the real exponential field. This subtheory thus provides a reasonable candidate for an axiomatization of the theory of the real exponential field. If one could prove that this subtheory is complete, then we would infer that the theory of the real exponential field is decidable.

Theorem 8.1 is a special case of a more general statement (appearing in [8]), giving necessary and sufficient conditions for a definably complete Baire expansion of an o-minimal structure by $\mathcal{C}^{\infty}$ functions to be o-minimal. More precisely, let $\mathbb{K}$ be a definably complete Baire structure, $\mathbb{K}_{0}$ be an o-minimal reduct of $\mathbb{K}$, expanding the field structure, and $\mathcal{F}$ be a family of total $\mathcal{C}^{\infty}$ functions definable in $\mathbb{K}$. We assume that $\mathcal{F}$ is closed under permutation of variables, contains the coordinate functions $\left(x_{1}, \ldots, x_{n}\right) \mapsto x_{i}$, and that if $f \in \mathcal{F}$, then $(\bar{x}, y) \mapsto f(\bar{x})$ is also in $\mathcal{F}$. Let $\mathbb{K}_{0}(\mathcal{F})$ be the reduct of $\mathbb{K}$ generated by $\mathbb{K}_{0}$ and $\mathcal{F}$. Let $\mathcal{G}_{0}$ be the set of all total continuous functions definable in $\mathbb{K}_{0}$, and $\mathcal{G}$ be the set of functions of the form $h \circ f$ for some $f: \mathbb{K}^{n} \rightarrow \mathbb{K}^{m}$ in $\mathcal{F}^{m}$ and some $h: \mathbb{K}^{m} \rightarrow \mathbb{K}$ in $\mathcal{G}_{0}$.

TheOREM 8.4 ([8, Theorem 6.2]). $\mathbb{K}_{0}(\mathcal{F})$ is o-minimal iff, for every $g \in \mathcal{G}$, there exists a natural number $N$ such that, for every affine set $A$, the set $V(g) \cap A$ has at most $N$ definably connected components.

However, a direct proof of Theorem 8.1 can be obtained by modifying the proof of [23, Theorem 1.9], which is the original statement of this result for expansions of the real field. Wilkie's proof uses measure-theoretic arguments. One can show that these arguments can be replaced by def-Baire category arguments. The reader familiar with [23] can check that Wilkie's proof goes through in our context as well, provided that one uses the following facts: 
(1) Every Charbonnel set is in $\mathcal{F}_{\sigma}$.

(2) For a Charbonnel set $A$ the following are equivalent: $A$ is meager, $A$ has empty interior, $A$ is nowhere dense.

(3) Every use of Fubini's Theorem in [23] can be replaced by the use of Proposition 5.4 .

(4) For Charbonnel sets, being meager is equivalent to being locally meager (cf. 3.9).

(5) Arguments in [23] involving sequences or topological compactness can be easily modified using definable compactness.

Acknowledgements. We would like to thank the referee for many useful observations. The second author was supported by the FCT grant SFRH/BPD/38523/2007.

\section{References}

[1] A. Berarducci and M. Otero, Intersection theory for o-minimal manifolds, Ann. Pure Appl. Logic 107 (2001), 87-119.

[2] J. Bochnak, M. Coste, and M.-F. Roy, Real Algebraic Geometry, Ergeb. Math. Grenzgeb. 36, Springer, Berlin, 1998.

[3] R. Cluckers and L. Lipshitz, Fields with analytic structure, J. Eur. Math. Soc., to appear.

[4] A. Dolich, C. Miller and C. Steinhorn, Structures having o-minimal open core, Trans. Amer. Math. Soc. 362 (2010), 1371-1411.

[5] L. van den Dries, Dense pairs of o-minimal structures, Fund. Math. 157 (1998), 61-78.

[6] L. van den Dries and P. Ehrlich, Fields of surreal numbers and exponentiation, ibid. 167 (2001), 173-188.

[7] A. Fornasiero, Tame structures, v. 3.0, preprint, 2009.

[8] A. Fornasiero and T. Servi, Theorems of the complement, v. 2.1, submitted, 2009.

[9] —, -, Relative Pfaffian closure of definably complete Baire structures, v. 2.2, submitted, 2010.

[10] S. Fratarcangeli, A first-order version of Pfaffian closure, Fund. Math. 198 (2008), 229-254.

[11] H. Gonshor, An Introduction to the Theory of Surreal Numbers, London Math. Soc. Lecture Note Ser. 110, Cambridge Univ. Press, Cambridge, 1986.

[12] V. Guillemin and A. Pollack, Differential Topology, Prentice-Hall, Englewood Cliffs, NJ, 1974.

[13] J. L. Kelley, General Topology, Van Nostrand, Toronto, 1955.

[14] A. G. Khovanskiŭ, Fewnomials, Transl. Math. Monogr. 88, Amer. Math. Soc., Providence, RI, 1991.

[15] A. Macintyre and A. J. Wilkie, On the decidability of the real exponential field, in: Kreiseliana, A K Peters, Wellesley, MA, 1996, 441-467.

[16] D. Marker, Khovanskii's theorem, in: Algebraic Model Theory (Toronto, ON, 1996), NATO Adv. Sci. Inst. Ser. C Math. Phys. Sci. 496, Kluwer, Dordrecht, 1997, 181193. 
[17] C. Miller, Expansions of dense linear orders with the intermediate value property, J. Symbolic Logic 66 (2001), 1783-1790.

[18] J. C. Oxtoby, Measure and Category. A Survey of the Analogies between Topological and Measure Spaces, Grad. Texts in Math. 2, Springer, New York, 1971.

[19] Y. Peterzil and C. Steinhorn, Definable compactness and definable subgroups of o-minimal groups, J. London Math. Soc. (2) 59 (1999), 769-786.

[20] A. Pillay and C. Steinhorn, Definable sets in ordered structures. I, Trans. Amer. Math. Soc. 295 (1986), 565-592.

[21] T. Servi, Noetherian varieties in definably complete structures, Logic Anal. 1 (2008), 187-204.

[22] A. J. Wilkie, Model completeness results for expansions of the ordered field of real numbers by restricted Pfaffian functions and the exponential function, J. Amer. Math. Soc. 9 (1996), 1051-1094.

[23] —, A theorem of the complement and some new o-minimal structures, Selecta Math. (N.S.) 5 (1999), 397-421.

Antongiulio Fornasiero

Institut für Mathematische Logik

Einsteinstr. 62

48149 Münster, Germany

E-mail: antongiulio.fornasiero@googlemail.com
Tamara Servi

Centro de Matemática e Aplicações Fundamentais Av. Prof. Gama Pinto 2 1649-003 Lisboa, Portugal E-mail: tamara.servi@googlemail.com

Received 23 April 2009;

in revised form 8 June 2010 